\title{
Energy storage for improvement of wind power characteristics
}

\section{Rasmussen, Claus Nygaard}

Published in:

IEEE PES Trondheim PowerTech

Publication date:

2011

Link back to DTU Orbit

Citation (APA):

Rasmussen, C. N. (2011). Energy storage for improvement of wind power characteristics. In IEEE PES Trondheim PowerTech IEEE.

\section{General rights}

Copyright and moral rights for the publications made accessible in the public portal are retained by the authors and/or other copyright owners and it is a condition of accessing publications that users recognise and abide by the legal requirements associated with these rights.

- Users may download and print one copy of any publication from the public portal for the purpose of private study or research.

- You may not further distribute the material or use it for any profit-making activity or commercial gain

- You may freely distribute the URL identifying the publication in the public portal

If you believe that this document breaches copyright please contact us providing details, and we will remove access to the work immediately and investigate your claim 


\title{
Energy storage for improvement of wind power characteristics
}

\author{
C. N. Rasmussen, Member IEEE
}

\begin{abstract}
Results from simulation of the influence of energy storage on the variability and availability of wind energy are presented here. Simulations have been done using a mathematical model of energy storage implemented in MATLAB. The obtained results show the quality improvement, of energy delivered by a combination of wind and energy storage, in relation to the size of the energy storage. The introduction of storage enables suppression of wind power fluctuations up to a timescale proportional to the storage energy capacity. Energy storage cannot provide availability of wind power at all times, but it can guarantee that a certain fraction of average wind power will be available within a given timeframe. The amount of storage energy capacity necessary for significant improvement of wind power availability, within a given period, is found to be approximately $20 \%$ of the energy produced in that period. The necessary storage power is found to be approximately equal to the average wind power of that same period.
\end{abstract}

Index terms-- Energy storage, Energy management, Wind energy, Power systems, Power system stability, Simulation

\section{INTRODUCTION}

Wind power is a rapidly growing and very promising renewable source of electric energy. But with a large fraction of wind power in the electric power system, the stochastic nature of wind power will start to play a significant role. A controllable and non-fluctuating supply is needed to fully secure availability, but this cannot be achieved with wind power alone.

Introducing an energy storage element in relation to a wind power plant changes the spectrum and statistical distribution of the output power. A gradual increase in the amount of storage (power and energy) associated with a wind power plant, will make the output more controllable, predictable and less variable.

A variety of energy storage technologies with diverse properties and attributes are available. In order to determine, which storage technologies that are most relevant in connection to wind power the required power- and energy levels of storage must be determined. The aim of using energy storage is to improve the overall quality of wind energy, but the actual improvement will always be subject to an objective judgment.

Storage technologies and their ability to create actual value in terms of production cost reductions have been analyzed in various ways, [9], [10], [11] and [12]. The outcome of such an analysis depends on a large number of parameters and assumptions. It therefore seems relevant to try to obtain some

C.N. Rasmussen is Associate Professor at the Technical University of Denmark, Department of Electrical Engineering. DK-2800 Lyngby, Denmark. cnras@elektro.dtu.dk basic measures of the wind energy quality improving effect of a given amount of energy storage.

What is presented here is the result of an effort to determine the energy quality improving effects of energy storage on wind power, on medium timescales, from minutes to weeks. Three possible indicators of a general improvement in the quality of wind energy are:

- Availability improvement; increasing the likelihood of a certain amount of power being available in a given timeframe.

- Variability reduction; reduction of wind power fluctuation sizes and rates.

- Predictability improvement; reducing the difference between wind power forecast and actually obtained wind power.

These ways of viewing the quality of wind energy will be explained and discussed later in section 6, but any parameter which may be perceived as a measure of improvement could in principle be used as measuring stick for the measurement of wind energy improvement.

When viewing the quality of wind energy from one of these three perspectives it is possible to quantify the term "quality" and establish a connection between the amount of storage (power and energy) and the obtained quality improvement.

In order to evaluate the whole concept of energy storage in relation to wind energy it is important to know how much storage that is needed to obtain the desired effect. Such information can be used to help choose the most optimal storage technology.

The analysis performed here has been done from a purely technical point of view, which means that the power marked is not considered. The reason for leaving out market issues is to be able to obtain more clear and straightforward results regarding the physical relations between energy storage and wind energy.

\section{ENERGY STORAGE TECHNOLOGIES}

A variety of technologies are available for storage of energy in the power system [18]. When identifying the most relevant storage solutions it is necessary to include considerations on many relevant parameters, such as: cost, lifetime, reliability, size, storage capacity and environmental impact. All these parameters should be evaluated against the potential benefits of adding storage in order to reach a decision on which type of storage should be added. There may also be cases where the value of adding storage is not large enough to justify such an investment. 
Energy storage technologies for power applications can be divided according to the form of energy stored: Mechanical, electro-chemical, electromagnetic, or thermal storage. Mechanical storage includes pumped hydro storage, compressed air energy storage (CAES) and flywheels. Electrochemical storage includes all types of batteries and fuel cells, and electromagnetic storage includes super capacitors and superconducting magnetic energy storage. Each technology has certain attributes with regard to for example storage capacity, power, reaction time and cost.

Fig. 1 shows some of the most relevant storage technologies, grouped according to the form of stored energy as well as energy storage capacity. The medium capacity storage technologies seem very relevant for storage in relation to wind power. The medium capacity storage technologies are primarily batteries and flow batteries, which all have the advantage in relation to wind power plants that they are modular and scalable.

In the low capacity end, ultra capacitors may be of relevance in relation to wind power conditioning. Hydrogen fuel cells may also be relevant, both as medium- and high capacity storage.

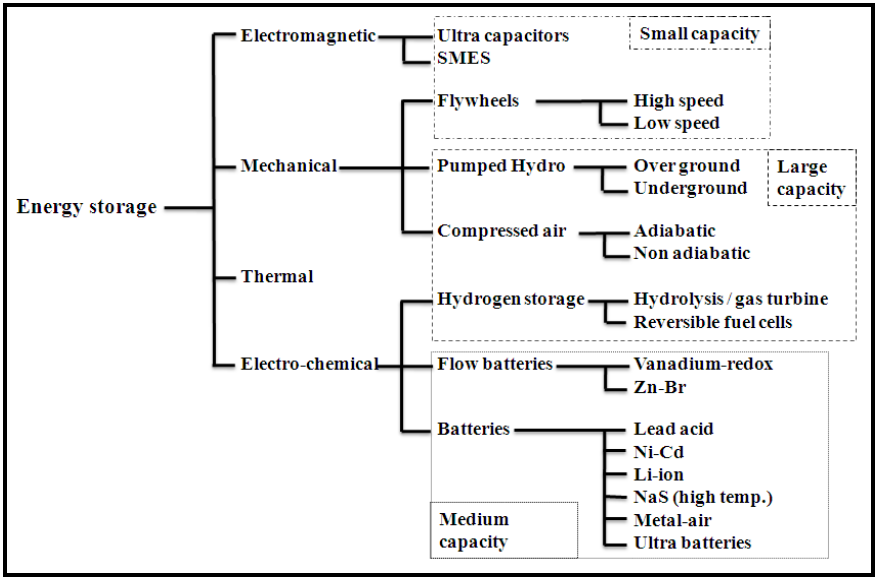

Fig. 1. Energy storage technologies grouped according to form of energy as well as energy storage capacity. Typical timescales have been indicated.

The low capacity storage technologies seem less relevant in relation to overall improvement of wind energy quality because of high cost pr. unit stored energy and relatively short storage time scale. The very high capacity technologies, pumped hydro storage and compressed air energy storage (CAES), involve large investments and civil engineering efforts, as well as special requirements with regard to placement. But these technologies may well be the best solution in relation to large scale storage, where a few major energy storage facilities ensure overall power system stability. Especially in cases where hydro-electric plants with unused storage capacity already exist, large benefits may be obtained by combining them with wind power plants [15].

\section{IMPROVEMENT OF WIND ENERGY QUALITY}

In order to obtain a clearer view of which kinds of improvements to power- and energy quality that an energy storage device can contribute with, some possible application areas are listed here:

\section{A. Spinning reserve and enhanced inertia}

Energy reserves equal to more than $15 \mathrm{~min}$ of supply allows for shutdown of smaller stand-by units such as local Combined Heat-and-Power (CHP) units. During periods of low load and high wind the storage will charge up to its maximum state of charge and stay in this state until a sudden reduction in available power occurs and reserves are needed.

Short term storage, of perhaps a few minutes of supply, can enhance the apparent inertial constant of the wind park and at the same time reduce short-term wind power variations.

\section{B. Peak shaving}

Peak shaving is removal or reduction of large deviations from average, on time scales from seconds to hours, depending on storage capacity.

\section{Wind power filtering}

A general reduction of wind power fluctuations can be obtained by running the energy storage as a kind of low-pass filter. The smoothening effect will depend on power and energy capacity of the storage, as well as the variability pattern of the wind. The time horizon for such a usage would stretch from seconds up to hours or days, depending on storage capacity [17]. In the simulations presented here, the availability increases and variability reductions are obtained by running the storage as a filter.

\section{Improving predictability}

Being able to meet a production forecast could be of large value. This would typically be a forecast of 12 to 48 hours ahead and the ability to follow the predicted production of such a forecast could be improved by the use of energy storage.

\section{E. Long-term load- and production leveling}

In case of large amounts of energy storage, long-term load or wind production leveling could be obtained. This would for example allow the use of wind farms as base load or enable an increase of predictability to nearly $100 \%$ for a certain period of time. The time horizon could be days or weeks, depending on storage size and the allowed variations. Such storage levels could also allow for shutdown of large stand-by reserves.

If the electric energy production is to be based primarily on renewable energy sources, a large amount of energy storage will have to be combined with overcapacity in generation.

Evaluating the actual value of any of the potential applications listed above, is difficult, since it involves market mechanisms and regulations special to each individual country, transmission system operator and type of power generation. In order to limit the number of parameters, these considerations have been left out. Clearly, what is profitable in one particular situation may be irrelevant in other cases, but considerations on how much storage that is needed in order to ensure a certain supply level are valid in general. In [9] the economic and environmental perspectives of energy storage have been considered for the specific case of storage in the Dutch electricity supply. In [12] an analysis of feasibility of various energy storage technologies has been carried out. A large number of parameters need to be included in such an analysis of economic value and this makes it very different 
from the analysis of effects of physical storage parameters presented here.

\section{THE NATURE OF WIND POWER VARIATIONS}

Wind power production $\left(P_{w}\right)$ varies with wind speed $(v)$ according to [3]:

$$
P_{w}(v) \propto v^{3}
$$

Typical minimum, nominal and maximum wind speeds are, $v_{\min }=4 \mathrm{~m} / \mathrm{s}, v_{n}=13 \mathrm{~m} / \mathrm{s}$ and $v_{\max }=25 \mathrm{~m} / \mathrm{s}$. The wind speed varies in a stochastic manner, with the statistical distribution of wind speeds being close to that of a Weibull distribution [1], [2], [5]. The statistical distribution of wind power differs somewhat from that of wind speed, while still resembling a Weibull distribution.

Different wind sites experience different statistical distributions of wind speed. The wind sites can be divided into classes according to average and standard deviation of the wind speed [5]. Table I shows the average wind speed $(\mu)$, standard deviation $(\sigma)$ and turbulence intensity factor $I_{\mu}=(\sigma / \mu)$ for 4 different wind classes.

TABLE I

STATISTICAL PARAMETERS FOR 4 IEC WIND CLASSES USED FOR THE MODEL RESULTS PRESENTED HERE [5].

\begin{tabular}{|l|c|c|c|c|}
\hline Class \# & 1 & 2 & 3 & 4 \\
\hline$\mu[\mathrm{m} / \mathrm{s}]$ & 10 & 8.5 & 7.5 & 6 \\
\hline$\sigma[\mathrm{m} / \mathrm{s}]$ & 1.63 & 1.49 & 1.4 & 1.26 \\
\hline$I_{\mu}$ & 0.163 & 0.175 & 0.187 & 0.21 \\
\hline
\end{tabular}

The statistical parameters do not define the variation spectrum. The performance, as well as required power and storage capacity, of an energy storage is strongly influenced by the variation spectrum, which means that knowledge of this is essential.

Wind variations are generated by atmospheric phenomena which take place on many orders of magnitude, with regard to both space and time [3]. The scales of these variations range from local turbulence on length scales of meters and timescales of seconds up to seasonal variations on global scales. It therefore seems reasonable to expect wind variations down to timescales of approximately $(d / \mu) \sim 10$ s, with $d \sim$ $100 \mathrm{~m}$ being the characteristic dimension of the wind generator and $\mu \sim 10 \mathrm{~m} / \mathrm{s}$ being the average wind speed. Very long term variations, with timescales up to 1 year, should also be expected. Variations in wind power on timescales below approximately $10 \mathrm{~s}$ will be attenuated due to the spatial smoothening effect of the turbine and wind turbine inertia.

An analysis of various sets of wind data shows that the wind spectrum has a strong degree of self-similarity, which is characteristic for fractals. Fig. 2 shows the power spectrum of a $2 \mathrm{MW}$ nominal power generator, on a time scale from 1 hour up to 2000 hours $\left(\sim 2 \frac{1}{2}\right.$ months). It was obtained by Fourier transforming a 2000 hour long vector with 1-hour interval measurements of power output from a wind generator.

There is a linear relationship between $\log (t)$ and $\log (P)$ which corresponds to a power-law relation:

$$
P(t)=t^{n}
$$

With $t=f^{-1}$ being the time scale. The slope $(n)$ of the line turns out to be $\sim 1$. According to [4], the case of $n=1$ corresponds to self-similarity with a fractal dimension of $D=$ 1.5. An analysis of other data sets shows that this relation continues at least down to $t \sim 6$ minutes, but the slope tends to decrease towards zero on timescales above $\sim 1 / 2$ year. A decreasing slope corresponds to larger fractal dimension $(D>1.5)$ and thereby a rougher appearance on larger scales.

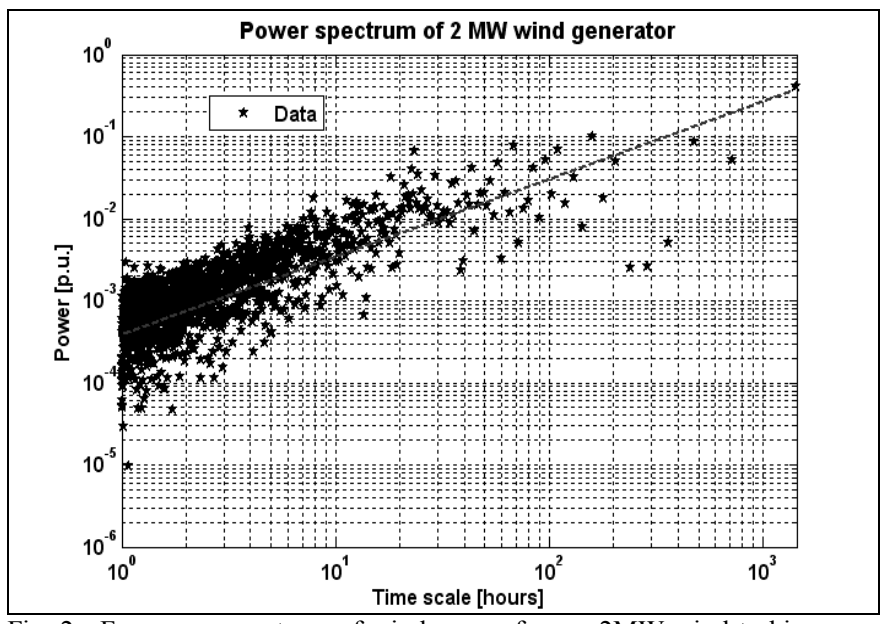

Fig. 2. Frequency spectrum of wind power from a $2 \mathrm{MW}$ wind turbine, on a timescale of $2000 \mathrm{~h}$. The line has a slope of 1 in the double-logarithmic plot.

A self-similar wind pattern means that a plot of wind power versus time appears similar on any time scale. This behavior of the wind points to a linear relationship, between required energy storage capacity and the time scale on which wind power is to be guaranteed or smoothened.

\section{ENERGY STORAGE MODELING}

When modeling the effect of energy storage on power quality, a set of storage properties and a control scheme of the storage must be chosen. For these simulations the amount of parameters has been kept as low as possible. The parameters include maximum storage power, energy capacity, charge and discharge efficiencies and preferred state of charge.

The basic equations define the relation between storage energy $\left(E_{s}\right)$ and power $\left(P_{s}\right)$ :

$$
\begin{aligned}
& \frac{\mathrm{dE}_{\mathrm{s}}}{\mathrm{dt}}=\frac{-\mathrm{P}_{\mathrm{s}}}{\varepsilon\left(\mathrm{P}_{\mathrm{s}}\right)} \text { for } \mathrm{P}_{\mathrm{s}}>0 \quad \frac{\mathrm{dE}_{\mathrm{s}}}{\mathrm{dt}}=-\varepsilon\left(\mathrm{P}_{\mathrm{s}}\right) \cdot \mathrm{P}_{\mathrm{s}} \text { for } \mathrm{P}_{\mathrm{s}}<0 \\
& \mathrm{P}_{\mathrm{s}}=\left(\mathrm{P}_{\text {req }}-\mathrm{P}_{\mathrm{w}}\right)+\frac{\left(\mathrm{E}_{\mathrm{s}}-\mathrm{E}_{\max } \cdot \mathrm{psoc}\right)}{\tau}
\end{aligned}
$$

With $\varepsilon=\varepsilon_{c}=\varepsilon_{d}$ being the charge or discharge efficiency, $P_{w}$ is the available wind power, $E_{\max }$ is the total energy storage capacity, psoc is the preferred state of charge and $\tau$ is a time constant related to recharge towards the preferred state of charge. When calculating the time it takes to reach preferred state of charge the efficiency needs to be included. $P_{\text {req }}$ is the total power required from the combination of wind and storage. This power requirement can be manually controlled or 
it can be continuously found using an algorithm. For these simulations the power requirement $P_{\text {reg }}$ at a given point in time is found as the average total power over a past period of length $\Delta t$ :

$$
\mathrm{P}_{\text {req }}(t)=\frac{1}{\Delta \mathrm{t}} \cdot \int_{t-\Delta t}^{t}\left(P_{w}+P_{s}\right) d t
$$

In this way the energy storage acts as a filter that aims to eliminate fluctuations on timescales below $\Delta t$. The ability to do this depends on wind power characteristics as well as the power and energy capacity of the storage.

The purpose of this simulation model, which has been implemented in MATLAB, has been to introduce different wind distributions and simulate the resulting power output from a combination of wind power and storage. By looking at the statistical distribution and variability of the combined output it is possible to evaluate the obtained improvement in energy quality, as function of storage energy and power.

Wind distributions can be found from actual sets of winddata or they can be generated artificially by using an algorithm [2], [6], which generates a temporal wind pattern with a specified average and standard deviation. Such an algorithm is in fact capable of producing a Weibull distributed wind pattern and the parameters can be set to imitate the desired wind class. The self-similar pattern with power spectrum according to (2) can also be reproduced with the algorithm. This seems to justify the use of a wind generation algorithm for making data sets to be used as input to the energy storage simulation model.

\section{SIMULATION RESULTS}

For these simulations, three ways of viewing wind energy quality improvement has been treated. The influence of storage power and energy capacity, on availability, variability and predictability, has been investigated. These properties of wind power are discussed in the following and the results are presented.

Looking at a wind power profile and the corresponding response of an energy storage device, as shown in fig. 3, gives an idea of how a storage facility, with control scheme governed simply by the physical relations stated in equations 4 and 5, will influence the total power output. In this case the storage efficiency is set to $\varepsilon_{f f}=100 \%$. The storage capacity is in this case equal to $1 \mathrm{~h}$ of nominal power, or $\sim 8 \%$ of the energy $E_{\text {tot }}$ produced in the given time period of 50 hours. This is due to the fact that the produced energy is app. 0.25 p.u. for 50 hours (=1 p.u. for 12.5 hours). The storage capacity of 1 p.u. in 1 hour is thus $1 / 12.5=8 \%$ of the total energy produced in the period. The storage power equals nominal power $\left(P_{n}\right)$ of the wind turbine. The effect of storage is to remove large power fluctuations and provide a response-delay.

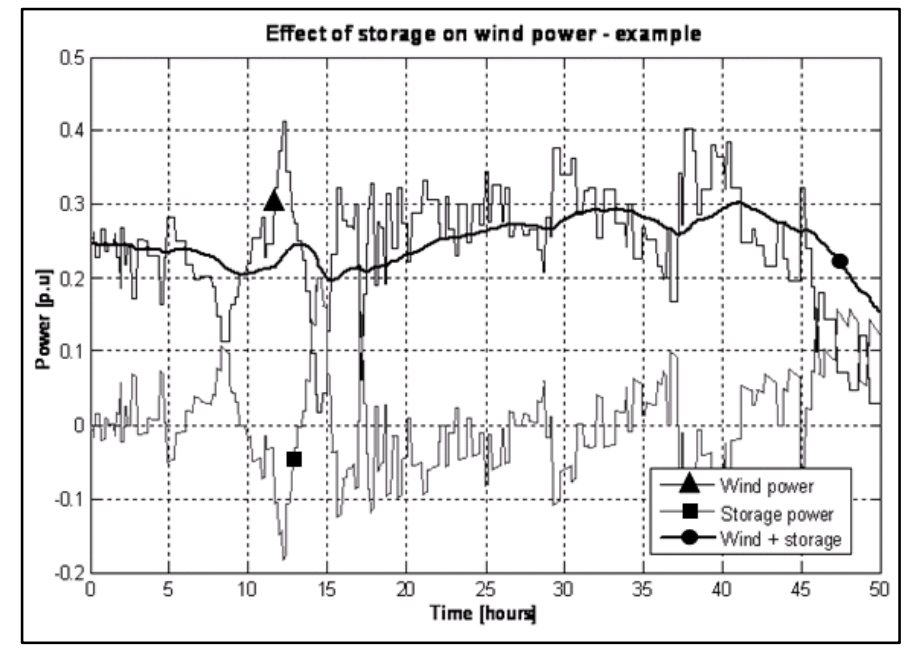

Fig. 3. The effect of introducing energy storage on total power output.

The statistical distribution, of the total power from the combination of wind and storage, is more uniform than that of wind power alone. Fig. 4 shows the change in cumulative distribution function of the power profile shown in fig. 3, from wind alone to wind and storage combined. Since $\varepsilon_{f f}=100 \%$ the average power does not change but the fraction of times with very high or very low power becomes smaller. Fig. 4 shows that with a storage capacity which is equal to $8 \%$ of the energy produced during the $50 \mathrm{~h}$ timeframe, a significant fraction of average power will be available with high probability. As an example: Without storage, only around $30 \%$ of the average power will be available $95 \%$ of the time period. When storage is included, almost $80 \%$ of the average power will be available $95 \%$ the period.

The influence of storage energy capacity, on the statistical distribution of output power, is shown in fig. 5. Increasing the storage capacity causes the probability density function to narrow in around average wind power for the given period. Average wind power is the maximum power that can be maintained for the entire period, no matter how large the storage capacity becomes. But with the introduction of storage it becomes possible to obtain any distribution within the limits set by the storage energy and power level and by the total energy production of the period. 


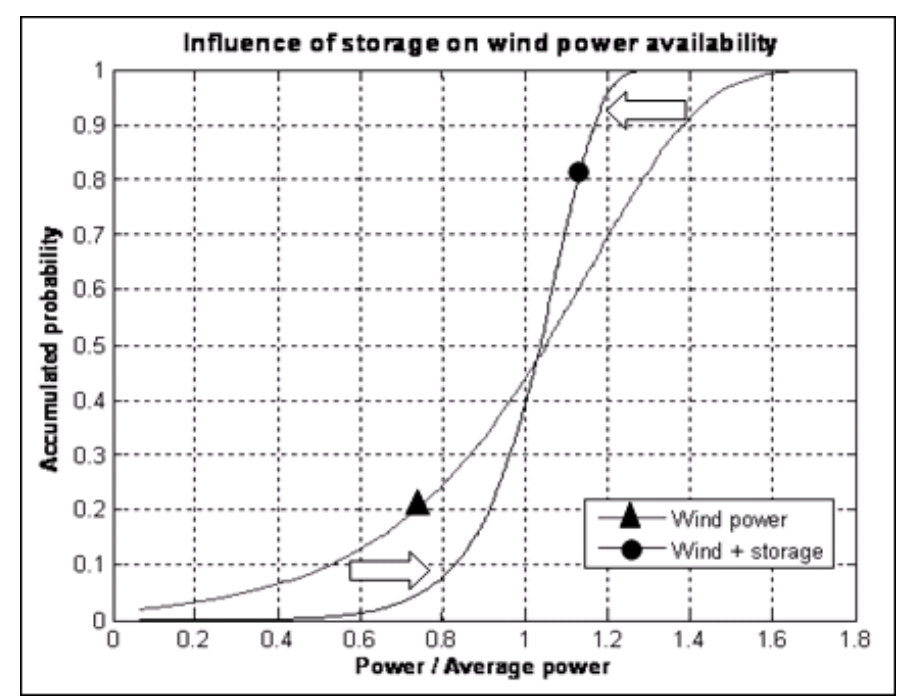

Fig. 4. Change in cumulative distribution of power output as a result of introducing energy storage in relation to wind power. The storage power is equal to nominal wind power $\left(\mathrm{P}_{n}\right)$ and storage energy capacity is equal to $8 \%$ of $E_{t o t}$.

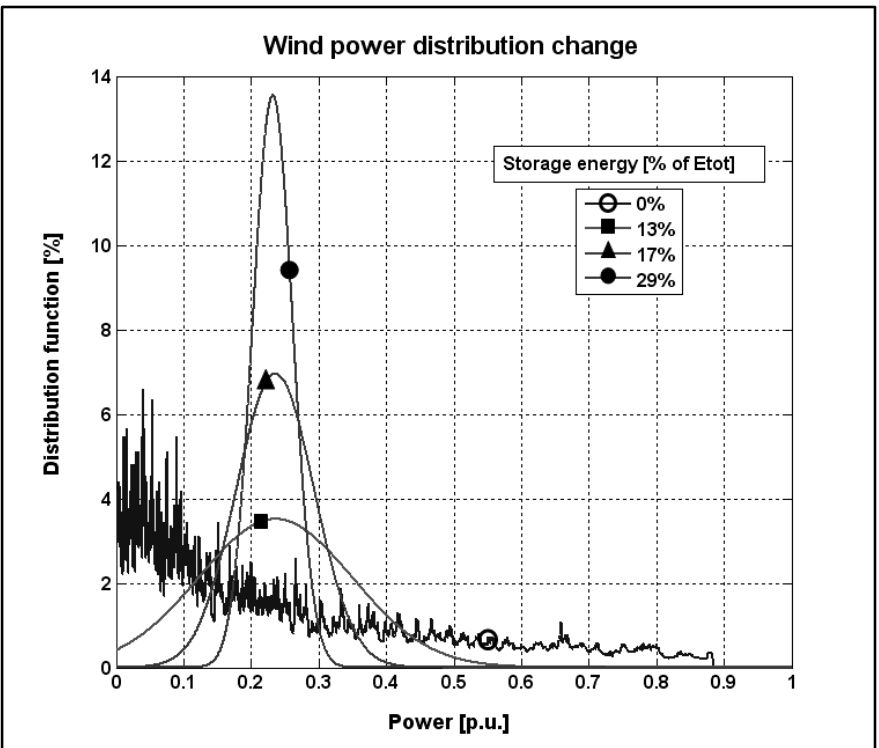

Fig. 5. Change in statistical distribution of power output from the combination of wind and storage as a result of increasing the energy storage capacity. With storage power $P_{n}$.

\section{A. Availability increase}

A relatively larger fraction of average power in a given time frame can be made available with a certain probability if storage is introduced. Power, energy capacity and efficiency of the storage will determine how large a fraction of average power that will be available. It is important to acknowledge that no amount of power will be available at all times. The level of availability refers to any specific period $\Delta t$ in which the average power is $\mathrm{P}_{\text {avg }}$ and the produced energy is $E_{t o t}=P_{a v g} \cdot \Delta t$. Because of the self-similar behavior of the wind pattern, the length of the time interval $\Delta t$ does not influence the levels of availability when plotted as function of energy in units of total energy produced and with power in units of average power of the period.

The wind patterns used for availability calculations have been made using the previously described algorithm, thus allowing for creation of wind profiles with a variety of statistical power distributions. The charge efficiency $\varepsilon_{c}$ and discharge efficiency $\varepsilon_{d}$ were both set to $85 \%$. The total efficiency of wind and storage combined may be calculated as:

$$
\varepsilon_{\mathrm{ff}}=\left(1-\mathrm{f}_{\mathrm{S}}\right)+\mathrm{f}_{\mathrm{S}} \cdot \varepsilon_{\mathrm{c}} \cdot \varepsilon_{\mathrm{d}}
$$

In eq. $6, f_{s}$ is the fraction of generated energy that passes through the storage. Assuming $\mathrm{f}_{s} \approx 1 / 2$ results in $\varepsilon_{f f} \approx 86 \%$. Average power out $\left(P_{\text {avgout }}=\varepsilon_{f f} P_{\text {avg }}\right)$ is the average wind power production minus the average power losses in the energy storage device.

Availability is here defined as the fraction of average power out $\left(\mathrm{P}_{\text {avg_out }}\right)$ that is available with a certain probability within a given period $\Delta t$.

The results show, that in order to be able to obtain a situation where a fraction of average power $P_{\text {avg }}$ is available during the period $\Delta t$, with a certain probability $(x \%)$, the storage energy capacity must be a certain fraction of the energy produced in that period $E_{t o t}=P_{\text {avg }} \cdot \Delta t$, and storage power must be a certain fraction of average power. Fig. 6 shows a contour plot of how much total power (wind + storage) that will be available during $95 \%$ of the time, for a class 1 wind distribution (strong average wind).

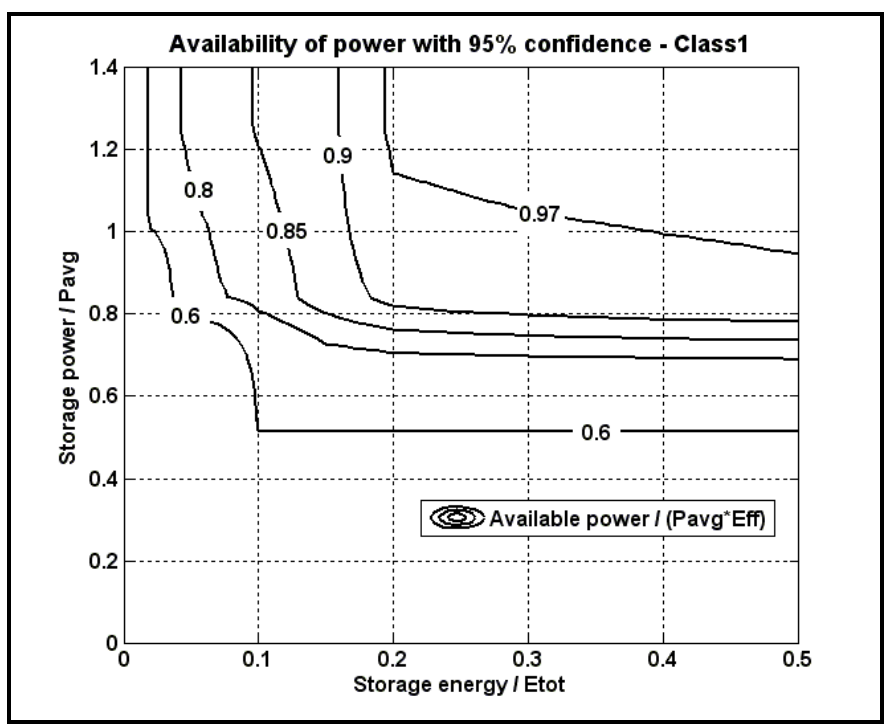

Fig. 6. Total power available with $95 \%$ confidence, as function of storage power and energy capacity, in any time period $\Delta t$ with average wind power $P_{\text {avg }}$ and total wind energy production of $E_{t o t}=P_{\text {avg }} \Delta t$. The available power is in units of average power out $\left(\mathrm{P}_{\text {avg_out }}\right)$. Wind class-1 distribution (defined in table I).

From fig. 6 it can be deducted that with an energy capacity of $20 \%$ of $E_{\text {tot }}$ it is possible to provide $>90 \%$ of average power out $\left(\mathrm{P}_{\text {avgout }}\right)$ with $95 \%$ confidence, provided that the storage power is larger than $80 \%$ of average wind power $\left(P_{\text {avg }}\right)$.

The average wind speed does not influence availability because of the dimensionless definition. But increasing the turbulence intensity factor (making the wind fluctuate relatively more) has the effect of increasing the storage energy capacity required to obtain the same level of availability, as it can be seen when comparing figures 6 and 7 . 
The actual amount of energy produced is much smaller for a class- 4 site than for a class- 1 site, but the required amounts of energy storage capacity are similar for the two wind classes. In order to guarantee $>90 \%$ of $\mathrm{P}_{\text {avg out }}$ with $95 \%$ confidence in a class-4 site, the required energy storage capacity is approximately $40 \%$ of $E_{\text {tot }}$ and the required storage power is $90 \%$ of $P_{\text {avg. }}$.

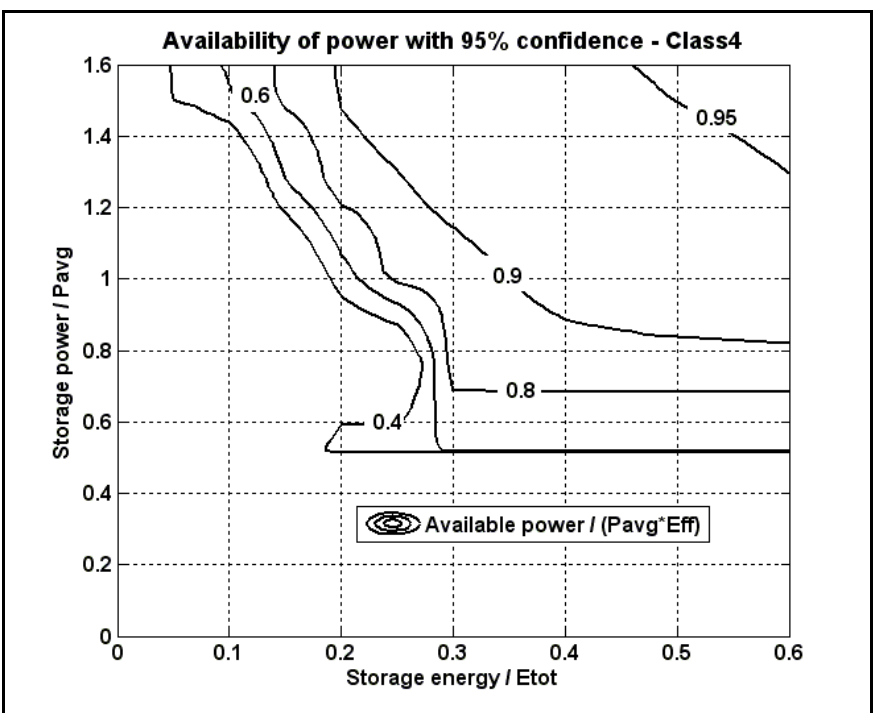

Fig. 7. Total power available with $95 \%$ confidence, as function of storage power and energy capacity, in any time period $\Delta t$ with average wind power of $P_{a v g}$ and total wind energy of $E_{t o t}=P_{a v g} \cdot \Delta t$. Wind class- 4 distribution (defined in table I).

A storage device can therefore not be made to guarantee anything at all times, but within a given timeframe the storage device can guarantee a fraction of the power average for that timeframe. This fraction depends on energy capacity, power and efficiency of the storage. Storage can thus be used, for example, to reduce the change rates in power from additional power generation equipment, such as CHP-plants, or to provide a running-delay that can ensure a certain amount of power for a specified time into the future.

If, for example, the requirement for a $1 \mathrm{MW}$ wind turbine placed at a class- 1 site, is to provide $90 \%$ of average power during $95 \%$ of a given 30-minute period, then the required energy and power of the storage can be found from fig. 6 .

Assuming that the average power of the 30 -min period is $50 \%$ of $\mathrm{P}_{n}=500 \mathrm{~kW}$ then the required storage energy is equal to $\mathrm{E}_{s}=0.2 \cdot 0.5 \mathrm{~h} \cdot 500 \mathrm{~kW}=50 \mathrm{kWh}$ and the required power is approximately equal to $\mathrm{P}_{\mathrm{s}}=0.8 \cdot 500 \mathrm{kWh}=400 \mathrm{~kW}$.

Table II shows the required energy capacity and power level for a storage device placed in relation to a $1 \mathrm{MW}$ wind generator, at two different sites.
TABLE II

REQUIRED STORAGE POWER $\left(P_{s-r e q}\right)$ AND ENERGY $\left(E_{s-r e q}\right)$ FOR A STORAGE DEVICE PLACED IN RELATION TO A 1MW WIND GENERATOR, AT TWO DIFFERENT SITES. WIND CLASSES ARE DEFINED IN TABLE I.

\begin{tabular}{|l|c|c|}
\hline Timeframe $(\Delta t)$ & $30 \mathrm{~min}$ & $30 \mathrm{~min}$ \\
\hline Confidence & $95 \%$ & $95 \%$ \\
\hline Availability & $90 \%$ & $90 \%$ \\
\hline Average wind power & $500 \mathrm{~kW}$ & $500 \mathrm{~kW}$ \\
\hline Wind class \# & 1 & 4 \\
\hline$E_{\text {tot }}[\mathrm{kWh}]$ & 250 & 250 \\
\hline$E_{\text {s-req }}[\mathrm{kWh}]$ & 50 & 100 \\
\hline$P_{\text {s-req }}[\mathrm{kW}]$ & 400 & 450 \\
\hline
\end{tabular}

The required storage energy and power, for smoothening with a 30 -min timeframe, is shown in the two bottom rows. Sites with strong stable wind, requires relatively less storage than sites with weak and variable wind. The simulations show, that ensuring availability of wind power on a time scale of $\Delta t$ requires storage energy capacity of approximately 20 to $40 \%$ of the energy produced within $\Delta t$ and power of approximately 80 to $100 \%$ of the wind power average during $\Delta t$. For smoothening to be effective on longer timescales, the storage energy $E_{s}$ and power $P_{s}$ must therefore be:

$$
\begin{array}{lll}
\mathrm{E}_{\mathrm{S}}(\Delta \mathrm{t})=\alpha \cdot \mathrm{C}_{\mathrm{f}} \cdot \mathrm{P}_{\mathrm{n}} \cdot \Delta \mathrm{t} & ; & \alpha \in[0.2 ; 0.4] \\
\mathrm{P}_{\mathrm{S}}=\beta \cdot \mathrm{C}_{\mathrm{f}} \cdot \mathrm{P}_{\mathrm{n}} & ; & \beta \in[0.8 ; 1]
\end{array}
$$

With $C_{f}$ being the capacity factor which is defined as average power divided by nominal power, $\mathrm{C}_{\mathrm{f}}=P_{\text {avg }} / P_{n}$. $C_{f}$ will typically be in the range of 0.2 to 0.4 for the wind classes considered here. If $C_{f}$ was to approach 1 , the resulting wind power output would be very stable because it cannot exceed $P_{n}$. This means that the validity of eq. 7 only holds for values of $C_{f}$ below approximately $C_{f} \sim 0.5$, above this point the required storage energy capacity begins to decrease.

These results cover wind variations corresponding to wind classes I to IV as defined in table I. Wind patterns which fall outside these limits will demand different levels of storage. A wind site with strongly fluctuating low winds would fore example demand storage energy above $40 \%$ of $\mathrm{E}_{\text {tot }}$ in order to ensure high availability.

A study of energy storage in relation to a wind power plant in Taiwan [7] indicated storage requirements of $E_{s} \approx 0.17$ $0.27 \cdot E_{\text {tot }}$ and $P_{s} \approx 0.46-1.1 \cdot \mathrm{P}_{\text {avg }}$ to obtain complete power leveling in a given period $\Delta t$. This is in good agreement with the results obtained here. In [14] and [16], the issue of securing power demand by the use of energy storage has also been treated and results that are consistent with the findings presented here were obtained.

The obtained results indicate that the relations stated above are valid on timescales ranging from hours to months. For shorter timescales they are likely to remain at least partially valid down to timescales of $\sim 10$ s and on longer timescales the validity is likely to hold up to approximately 1 year. 


\section{B. Variability reduction}

Variability can for example be defined as the maximum deviation from average power in a given period $\Delta \mathrm{t}$. Or it can be defined as the maximum deviation from the power available at the beginning of the period. In some cases it may be most relevant to look at the negative power deviations, which are the reductions in available power relative to the average. When applying this viewpoint there is not much difference between availability and negative variability. Variability is given as the standard deviation of the statistical power distribution. As figures 4 and 5 show, the effect of adding storage is to reduce the standard deviation and thereby make a larger fraction of average power available. In Fig. 8, simulated levels of variability at a class-4 wind site are shown.

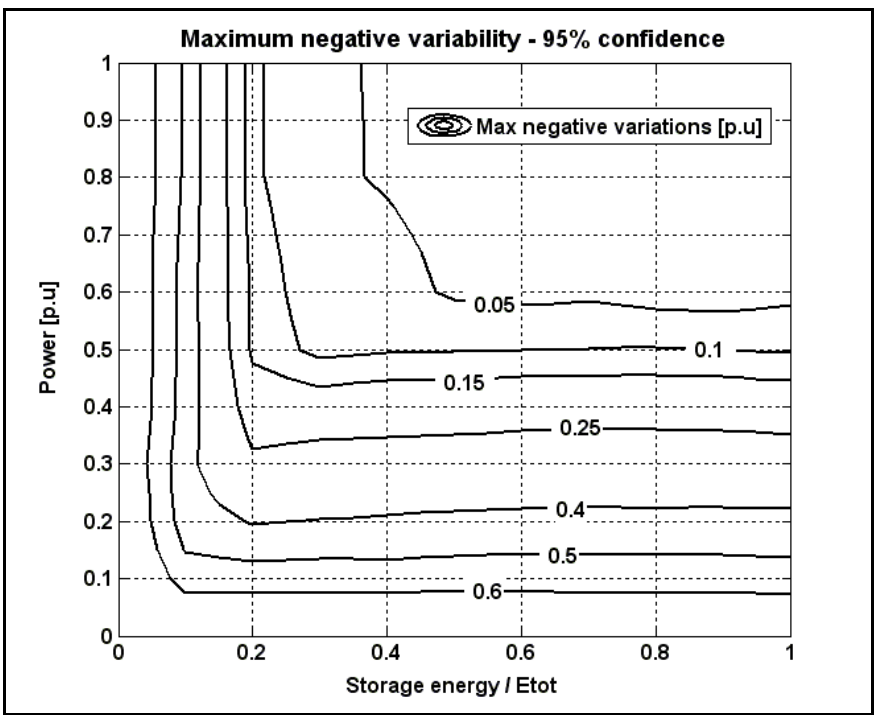

Fig. 8. Obtained reduction in wind power variability (within a time-frame of 1 hour) with energy storage. Largest deviations from average power at a class4 wind site (wind sites are defined in table I).

With storage power equal to $50 \%$ of nominal wind power and storage energy equal to $20 \%$ of $E_{\text {tot }}$ the maximum variations of total power in negative direction can be kept below $15 \%$ of nominal power. The storage requirement for significant reduction in power variations is thus smaller than the requirement for a large increase in availability.

Just how much the standard deviation is reduced with increasing storage level is shown in fig. 9. With relatively small storage energy capacity equal to $10 \%$ of $E_{\text {tot }}$ the power level of the storage only has little influence on the standard deviation. On the other hand, for a storage energy capacity of more than $40 \%$ of $E_{t o t}$, the power level of the storage becomes all-important as the energy capacity losses its importance. In the energy range of 10 to $40 \%$ of $E_{t o t}$ both storage energy and power have influence on the standard deviation of the power output.

This shows that a strong improvement in the quality of wind energy can be obtained by introducing energy storage with a capacity equal to approximately $10 \%$ of the energy produced on the required timescale and storage power equal to approximately average power production.

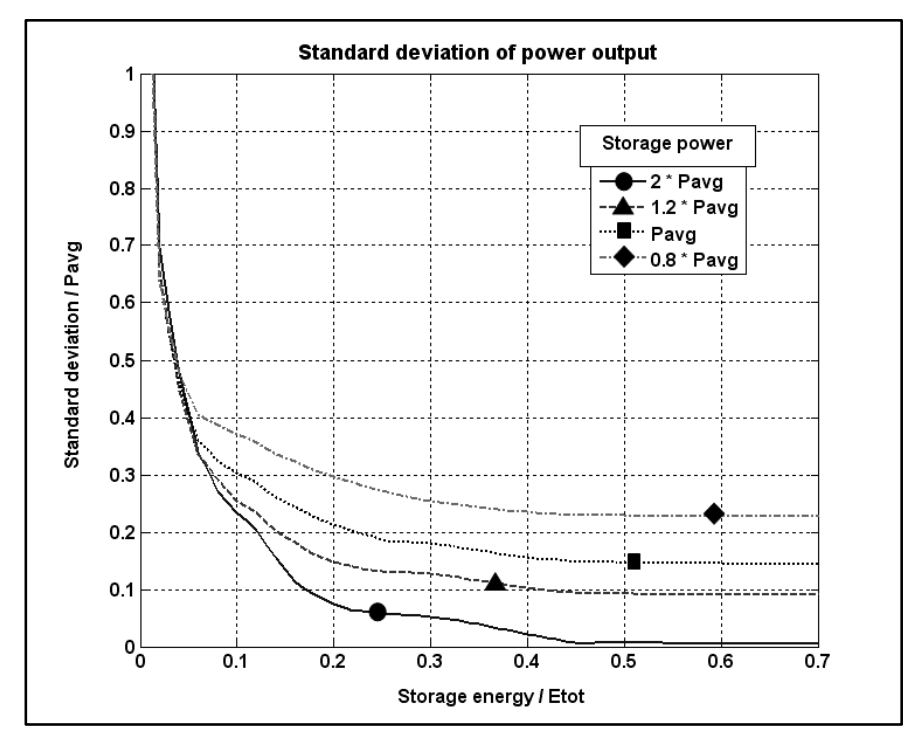

Fig. 9. Standard deviation of total output power from the combination of wind power and storage as a function of storage power and energy capacity. Data from a $2 \mathrm{MW}$ wind generator during a $2000 \mathrm{~h}$ period.

\section{Predictability improvement}

It may be required by owners of wind power plants that they provide a power generation forecast of for example 12 to 48 hours ahead. The accuracy of such a forecast could be improved with energy storage by absorbing a fraction of the deviation between forecast and actual production. The storage energy needed for this purpose also depends on the length of the timeframe for which wind power should be predicted, as well as the size and variation pattern of the error.

The forecast power error, which is the difference between forecast power and actual wind power, may be divided into a systematic and a random component [3]. The power required by a storage device in order for it to effectively reduce forecast errors will be comparable to the rms-value of the forecast error of power prediction $\left(\sigma_{r m s}\right)$. The storage must aim at compensating for the difference between actual and forecast power and the rms-value of the power required to do this must therefore be equal to the rms-value of the forecast error.

The required storage energy will largely depend on the systematic error $\left(\mu_{e}\right)$ since the random error will fluctuate around the systematic error on a shorter timescale and only add a small contribution to the storage requirement. Using data from [8], the rms-value of the forecast wind power error for the North Sea area is calculated to $\sigma_{r m s} \sim 0.2$ p.u. whereas the systematic error is found to be $\mu_{e} \sim 0.1$ p.u. This means that the energy capacity requirement, of energy storage used for forecast improvement, is in the order of $\sim 0.1 \cdot P_{n} \cdot \Delta t$, with $\Delta t$ being the length of the forecast timeframe. With a 36-hour forecast timeframe the energy requirement thus becomes equal to 3.6 hours of nominal power (or 3.6p.u. hours/0.2p.u. $=18$ hours of 0.2 p.u.). 


\section{CONCLUSIONS}

The quality of wind energy, defined here as availability, variability or predictability, may be strongly improved by introducing energy storage. The results obtained can be used to determine the relevant level of storage for a given timescale and size of the wind power plant. Ensuring a large fraction of average power, on timescales from minutes up to hours, seems obtainable with the use of for example battery storage.

The storage energy and power requirement, for significant availability improvement in a given period, is found to be 20 to $40 \%$ of the energy produced in the period and 80 to $100 \%$ of average power for the period. The storage power and energy requirements, for significant improvement of wind energy quality, for e.g. a $1 \mathrm{MW}$ wind generator, on a 30 minute timescale, are approximately $400 \mathrm{~kW}$ and $50 \mathrm{kWh}$.

For some battery types this may pose a potential problem for short timescale applications. Several types of batteries such as Lead-acid, Li-ion and $\mathrm{NaS}$ have a relatively fixed relation between power and energy rating, with minimum discharge time typically being in the range of 1 hour. This for example means that a battery with a maximum power of $400 \mathrm{~kW}$ has an energy capacity of typically $400 \mathrm{kWh}$. For short timescale applications the energy capacity of some battery types will therefore have to be overrated. This does not imply that batteries cannot be used for timescales below a few hours, but they will have to be oversized in terms of energy capacity or perhaps custom build to obtain high charge- and discharge rates. Flow batteries do not have a fixed relation between energy and power capacity and this seems to give them an advantage on the short to medium timescales compared to several other battery types.

Ensuring availability and reducing variability are two strongly coupled ways of looking at improvement. Making power more available means making it more predictable, reliable and controllable. Because of the stochastic and selfsimilar nature of wind power, ensuring any significant level of wind power at all times will demand extreme energy storage capacity in the range of $20 \%$ of the annual energy production.

But the prospect of energy storage is rather to remove fluctuations on shorter timescales (seconds to several hours) in order to reduce variability and perhaps to shift wind production from night to day in order to accommodate loadand wind production variations.

Predictability can be improved with storage and the required power is limited to perhaps $20 \%$ of nominal wind power. But timescales of $\sim 36$ hours result in large energy requirements unless small systematic prediction errors can be obtained.

\section{REFERENCES}

[1] Freris, L. L.: "Wind energy conversion systems". Prentice Hall, Nov 1990.

[2] Helle, L.: "Modeling and comparison of power converters for doubly fed induction generators in wind turbines". Aalborg University, April 2007.

[3] Fox, B., D. Flynn, L. Bryans, N. Jenkins, D. Milborrow, M. O’Malley, R. Watson \& O. Anaya-Lara: "Wind power integration - connection and system operational aspects". The institution of Engineering and Technology, 2007.
[4] Fox, C.G.: "Empirically derived relationships between fractal dimension and power law form frequency spectra”. Birkhäuser Verlag, Basel, 1989.

[5] DS/EN 61400-2: "Elproducerende vindmøller-2. Del". Danish standards, 2006.

[6] McIver, A.D., P. Freere \& D.G. Holmes: "Watts happening in wind energy - grid connection of a variable speed wind turbine using a matrix converter". Fuel and energy abstracts. Vol. 36. No. 4:274, July 1995.

[7] Lu, S.M., C. Chang, W. Lee \& L. Wang: "Combining the wind power generation system with energy storage equipments". IEEE Industry applications society annual meeting, 2008. ISBN: 978-1-4244-2278-4

[8] Hopkins, J.S.: "The accuracy of wind and wave forecasts". UK met office, 1997.

[9] Ummels, B., C., E. Pelgrum \& W. L. Kling: "Integration of large-scale wind power and use of energy storage in the Netherlands' electricity supply". IET Renewable Power Generation, Vol. 2. No.1. 2008.

[10] Spahic, E., G. Balzer, B. Hellmich \& W. Münch: "Wind energy storages - Possibilities". IEEE PowerTech, 2007.

[11] Hoffmann, C., M. Greiner, L.V. Bremen, K. Knorr, S. Bofinger, M Speckmann \& K. Rohrig: "Design of transport and storage capacities for a future European power supply system with a high share of renewable energies". Third International Renewable Energy Storage Conference, 2008 .

[12] Oberschmidt, J. \& M. Klobasa: "Economical and technical evaluation of energy storage systems". Third International Renewable Energy Storage Conference, 2008.

[13] Joerissen, L., J. Garche, C. Fabjan \& G. Tomazic: "Possible use of vanadium redox-flow batteries for energy storage in small grids and stand alone photovoltaic systems". Journal of power Sources, no. 127, 2004.

[14] Weissbach, R.S., R.E. Teodorescu \& J.R. Sonnenmeier: "Comparison of Time-Based Probability Methods for Estimating Energy Storage requirements for an off-grid residence". IEEE Energy 2030. Atlanta, USA, 2008.

[15] Elistratov, V V.: "Hybrid system of Renewable Energy Sources with hydro accumulation". Third International renewable energy storage conference (IRES) 2008, Eurosolar.

[16] Faias, S., J. Sousa \& R. Castro: "Evaluation of the Portuguese energy storage needs due to the increasing integration of renewable sources". Third International renewable energy storage conference (IRES) 2008, Eurosolar.

[17] C.N. Rasmussen: "Improving wind power quality with energy storage", IEEE conference on sustainable renewable energy, Valencia, Spain, 2009 - Conference proceedings.

[18] Swierczynsky, M., R. Teodorescu, C.N. Rasmussen, P. Rodriguez, H. Vikelgaard: "Storage Possibilities for Enabling Higher wind energy Penetration". EPE Wind Energy Chapter Symposium 2010, 15-16 April 2010, Stafford, UK.

\section{BIOGRAPHY}

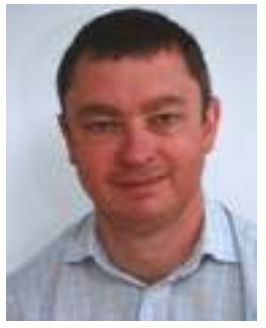

Claus Nygaard Rasmussen received a M.Sc. (eng) in 1997 and a $\mathrm{PhD}$ in 2004, from the Technical University of Denmark. He has worked with research and development within the areas of superconducting power cables, electrical insulation, magnetic field simulations, computer cooling and energy storage. He is now Associate Professor at the Technical University of Denmark. 